\title{
Research on Integration of Quality Testing based on Communication Technology
}

\author{
Meng-qing TANLI ${ }^{1,{ }^{*}}$, Yan JIANG $^{1}$, Yu-lin WANG ${ }^{1}$, Xiang WANG $^{1}$ \\ and Ru-shu PENG ${ }^{1}$ \\ ${ }^{1}$ School of Mech. \& Eng. University of South China, Hunan, Hengyang, China, 421001 \\ *TLMQ-TEAM@163.com
}

Keywords: Integration research, Communication technology, linterface communication.

\begin{abstract}
This paper prosposes an integration research to apply advanced communication technology in quality testing, including three aspects: integration of inspection function based on equipment, integration of inspection equipment based on interface communication, and integration of inspection unit based on computer communication. In order to give a clear illustration, an actual example with manufacturing factory is investigated.
\end{abstract}

\section{Introduction}

Measuring and testing technology plays a very important role in manufacturing technology. The general trend and guide post of quality testing are: (a) automation, (b) integration, and (c) digitalization, and integration technology in quality testing will be a new research region of quality testing technology.[1]

Research on integration technology of quality testing usually includes three aspects: (a) Integration of inspection function based on equipment, this is able to sum as testing function integration. (b) Integration of inspection equipment based on interface communication, this is called equipment integration. (c) Integration of inspection unit based on computer communication, this is also called computer integration. These aspects are mainly focused on integration of distinct function and information, but another integration of multi-sensor is mainly addressed in synonymous information. This research will investigate the former.[2,3,4]

In the past, the integration technology is focused on software integration, but the integration of hardware is also very important because it is the concrete base and carrier of integration technology.[5]

\section{Integration of Hardware in Quality Testing}

\section{Integration of Inspection Function based on Equipment}

In product testing, there are many quality indexes to inspect. If a testing equipment can inspect several parameters at one time, it will improve the efficiency and decrease the expensive of testing.

\section{Basic Principle of Function Integration in Finish Testing}

The inspection equipment is the apparatus and device for implementation measuring and testing function. Integration of inspection equipment usually implies that an inspection equipment can implement several functions in quality testing. Here, a typical 
example is illustrated in product quality testing, that is, testing apparatus of shape and position error. This apparatus can implement three functions including: (a) testing of radial error, (b) testing of axiality error, (c) testing of symmetry. Illustration of function integration of testing apparatus of shape and position error is shown in figure 1.

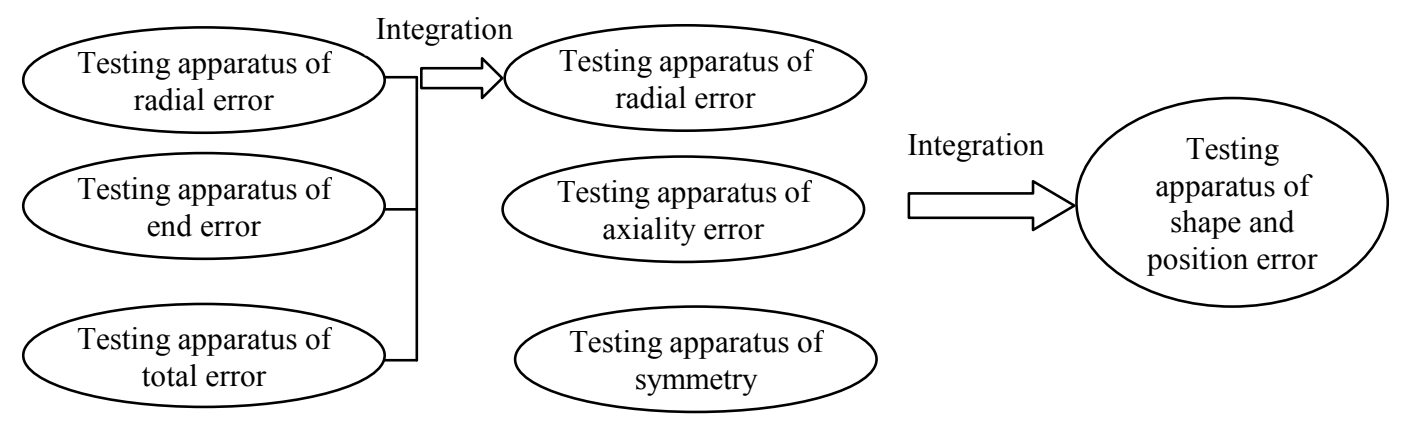

Figure 1 function integration — testing apparatus of shape and position error

\section{Hardware Implementation of Integration of Inspection Function}

The inspection equipment is the apparatus and device for realization of measuring and testing function. Integration of inspection equipment usually implies that an inspection equipment can implement several functions in quality testing. Here, a typical example is illustrated in product quality testing, that is, testing apparatus of shape and position error. This apparatus can implement three functions including: (a) testing of radial error, (b) testing of axiality error, (c) testing of symmetry. Hardware implementation of testing apparatus of shape and position error is shown in figure 2.
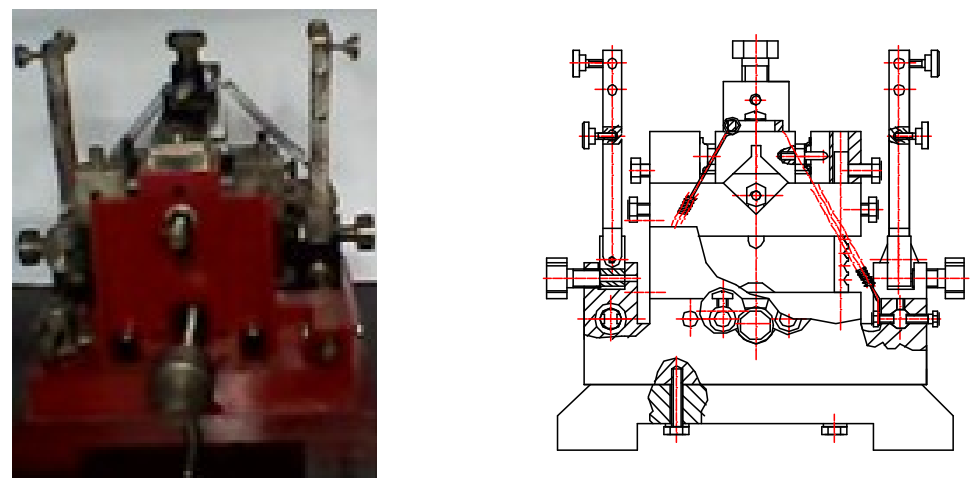

Figure 2 concrete objective - testing apparatus of shape and position error

\section{Integration of Testing Equipment based on Interface Communication}

With the rapid development of computer technology, the speed and performance of computer have improved exponentially. Computer has been used in quality testing deeply and widely, and several instruments can be carried to only one computer, this tends to the integration of testing equipment based on interface communication. Hence, how to connect the instrument and computer or what type of communication is adopted is becoming a very important technology. 


\section{Interface Communication in Quality Testing}

There are several types of communication between inspection device and computer, usual types are: RS232C, USB, and PCI. RS232C has become the interface standard between data terminal equipment and data communication equipment, the connector of RS232C has two profile: DB25 and DB9, and DB9 is usually applied in PC. USB is new interface communication standard of external device in computer, and has function of plug and play. PCI imply the slot in the motherboard of the computer. Detail is shown in figure 3.

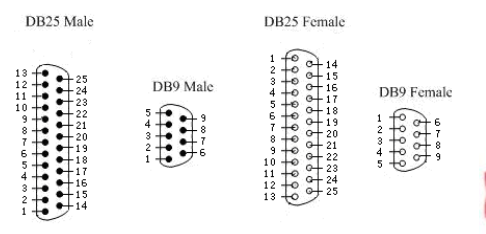

(a) RS232C

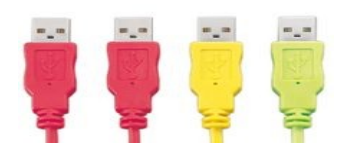

(b) USB

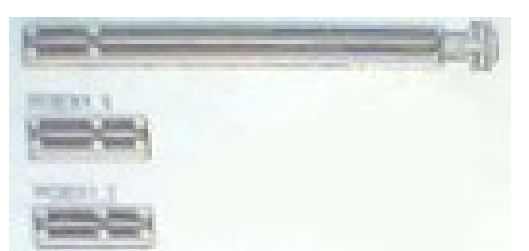

(c) PCI

Figure 3 usual types of communication between inspection device and computer

Every kind of interface has its communication format. For example,connection setup of RS232C for digital dial is described as table 1.

Table 1 Setup of interface communication RS232C for digital dial

\begin{tabular}{|l|l|l|l|}
\hline & Item & Value & Mem \\
\hline 1 & Port & COM1 & Fig. 3 (a) \\
\hline 2 & Pin type & DB9 & Fig. 3 (a) \\
\hline 3 & Baud & 1900 byte & Fig. 3 (a) \\
\hline 4 & CRC & odd & Fig. 3 (a) \\
\hline
\end{tabular}

\section{Arrangement of Testing Equipment based on Interface Communication}

In integration of testing equipment based on interface communication, suitable interface type should be choose according to concrete inspection instrument. Usually, RS232C is widely used in a lot of inspection device especially for older instrument, USB is applied in more new testing equipment, and PCI is generally used in data acquisition device developed by user self. For update microcomputer performance, RS232C , USB and PCI can not only be used singly, but also be used simultaneously. Illustration of integration of testing equipment based on interface communication is shown in figure 4 .

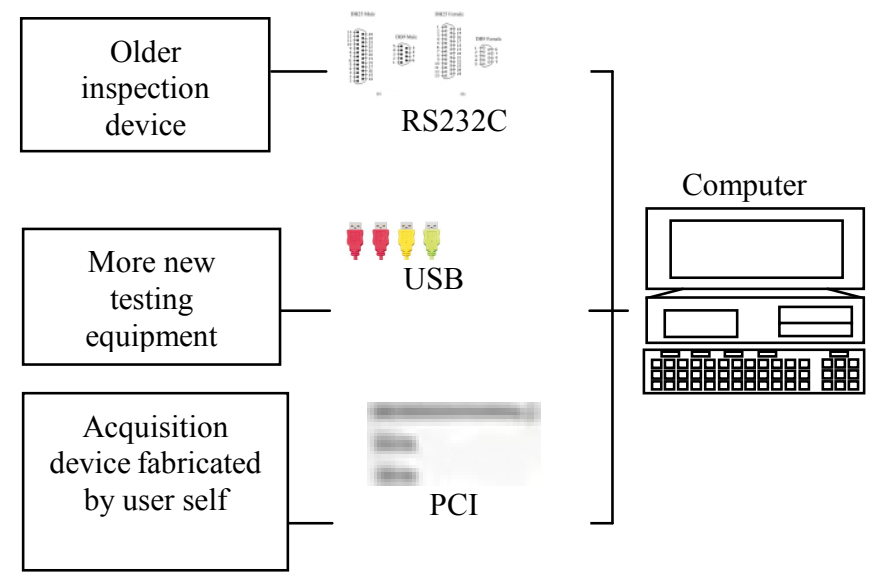

Fig.4 integration of inspection equipment based on interface communication 


\section{Integration of Testing Unit based on Computer Communication}

\section{Basic Principle of Integration based on Computer Communication}

Computer communication is most new technology for information technology and information era, especially Internet technology. Basic soul of computer communication is delamination and package, in which protocol is divided into several layers and transmitting data is packed into a series of data package. Based on this principle, logic structure of computer communication is descript as figure 5, the left is the description of Ethernet which is mainly applied in local network, and the right is the description of Internet which is mainly applied in communication among all kinds of computer network.

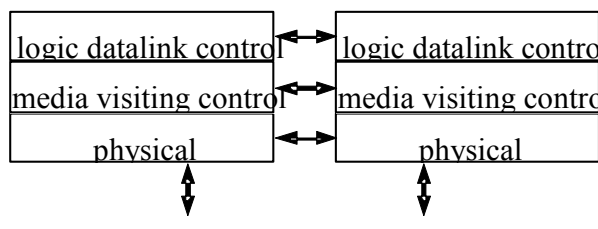

(a) Ethernet

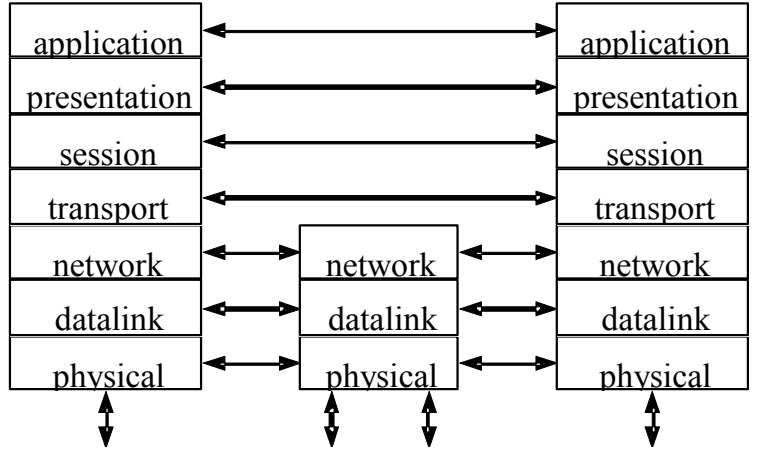

(b) Internet

Fig.5 Logic structure of computer communication

\section{Realization of Integration Based on Router}

Usually, integration of testing unit based on computer communication have two types: router and exchanger, but in quality testing, integration based on router is a more effective type. To realize computer network for quality testing, physical structure of computer communication integration based on router is shown as figure 6. For realization of integration based on router, following items should be careful:

(a) The router should be produced by famous manufacturer.

(b) The cable should have good shielding layer.

(c) Integrated communication plug for network is more effective other than network card.

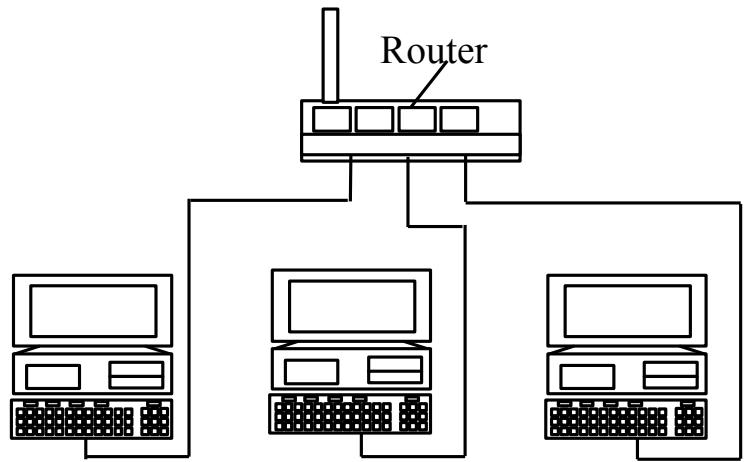

Figure 6 physical structure of computer communication 


\section{Software Integration of Quality Testing}

\section{Concrete scheme of Software Integration in Quality Testing}

Generally, software should include application integration and system integration, but application integration is the main topic in quality testing. The concrete scheme of application integration in quality testing is given by figure 7 , in this figure, SMCTW is the abbreviation of Shanghai Measuring \& Cutting Tool Works.

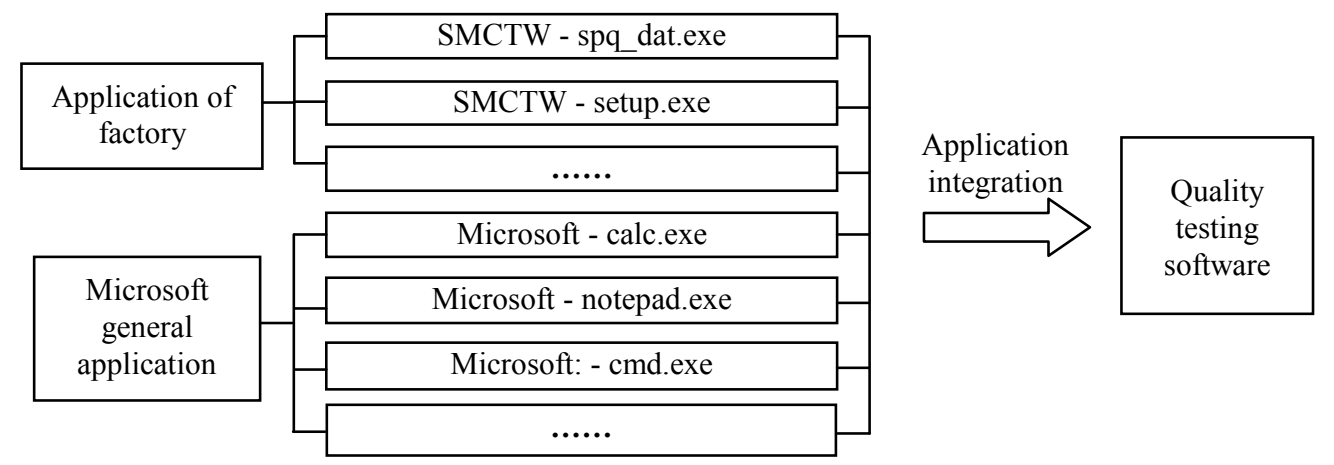

Figure 7 concrete scheme of software integration

\section{Modeling of Software Integration}

\section{Function Modeling of Software Integration with IDEF0}

IDEF0 (Integration DEFinition language0) is both semantics definition and comprehensive methodology for develop models consisted of functions and function relationship. The function analysis with IDEF0 uses top-down approach, and should begin at top-level context diagram called A_0, in which, the maximum abstract and general function are descript. The A_ 0 diagram of quality testing of integration with interface communication is shown in figure 8. And figure 9 has described functions at A0-level in detail.[6,7]

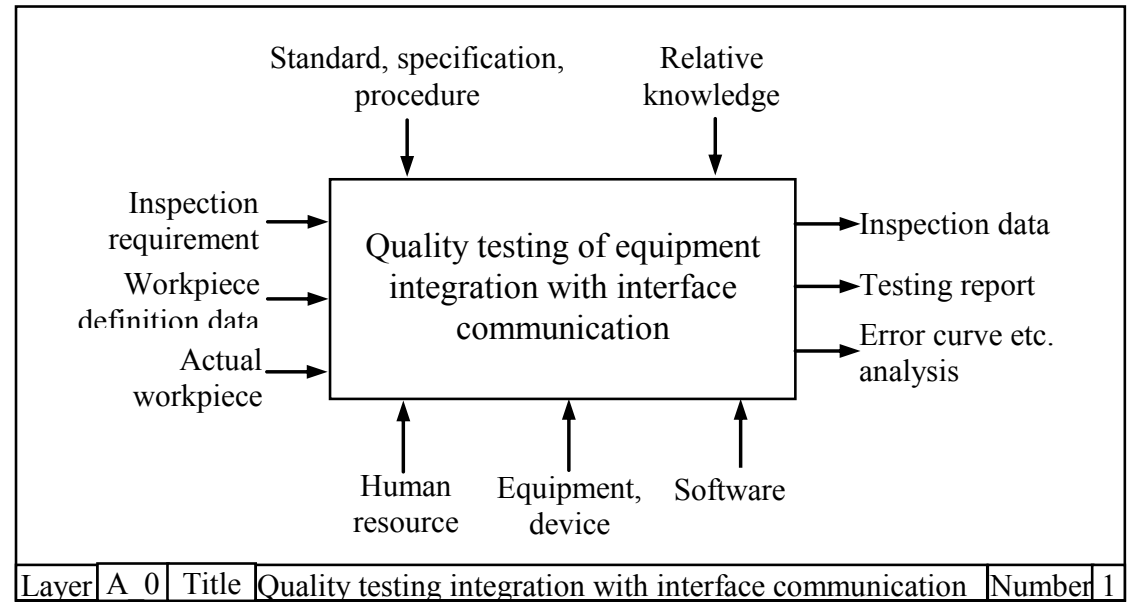

Figure 8 A_0 diagram of quality testing integration with interface communication 


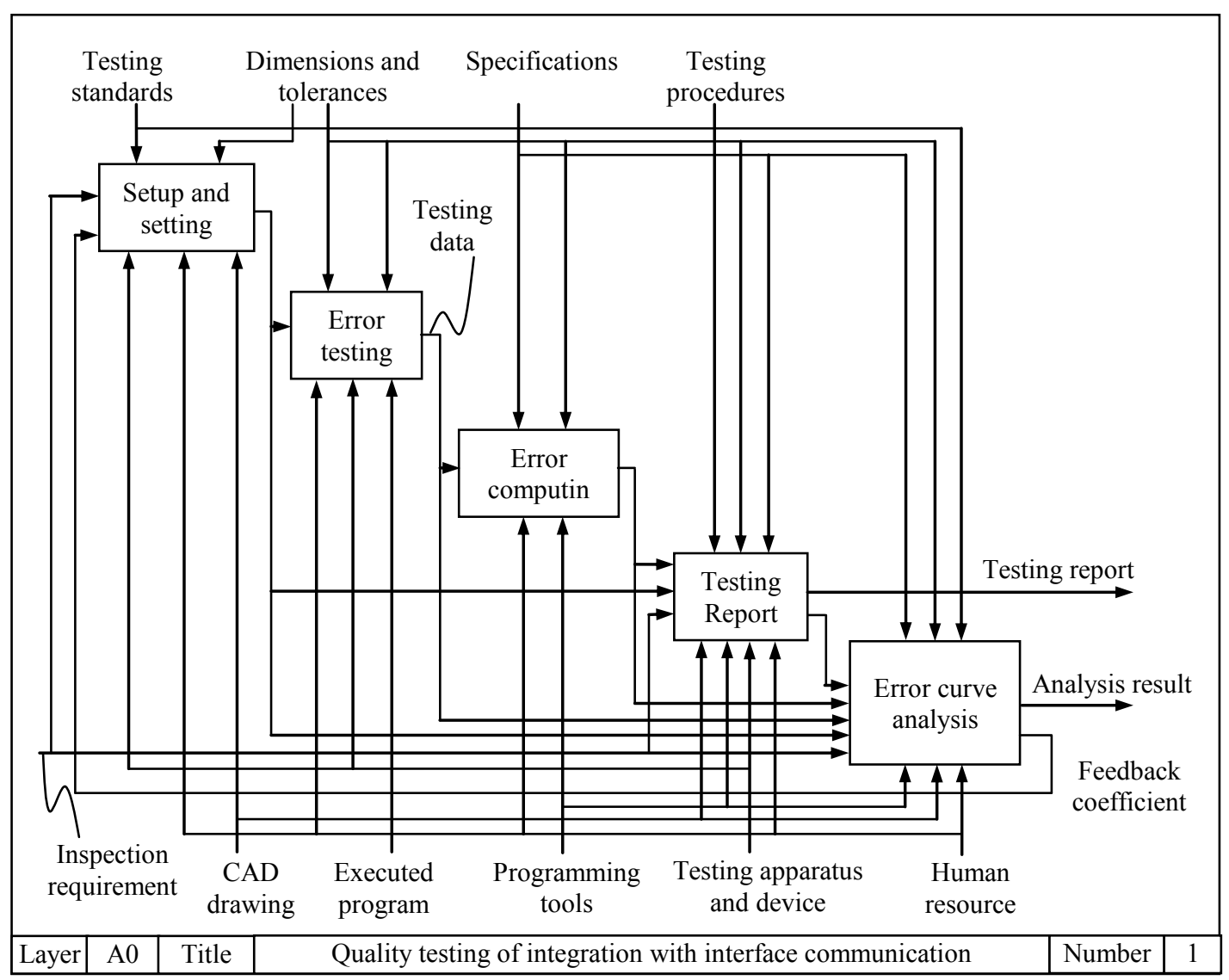

Figure $9 \mathrm{~A} 0$ diagram of quality testing integration with interface communication

\section{Information Modeling of Software Integration with Object-oriented Model}

Object-oriented model is an advanced modeling tool, information modeling of software integration in quality tseting can use object-oriented model. The class diagram as figure 10 has described the software class comprising including attributes and member functions.

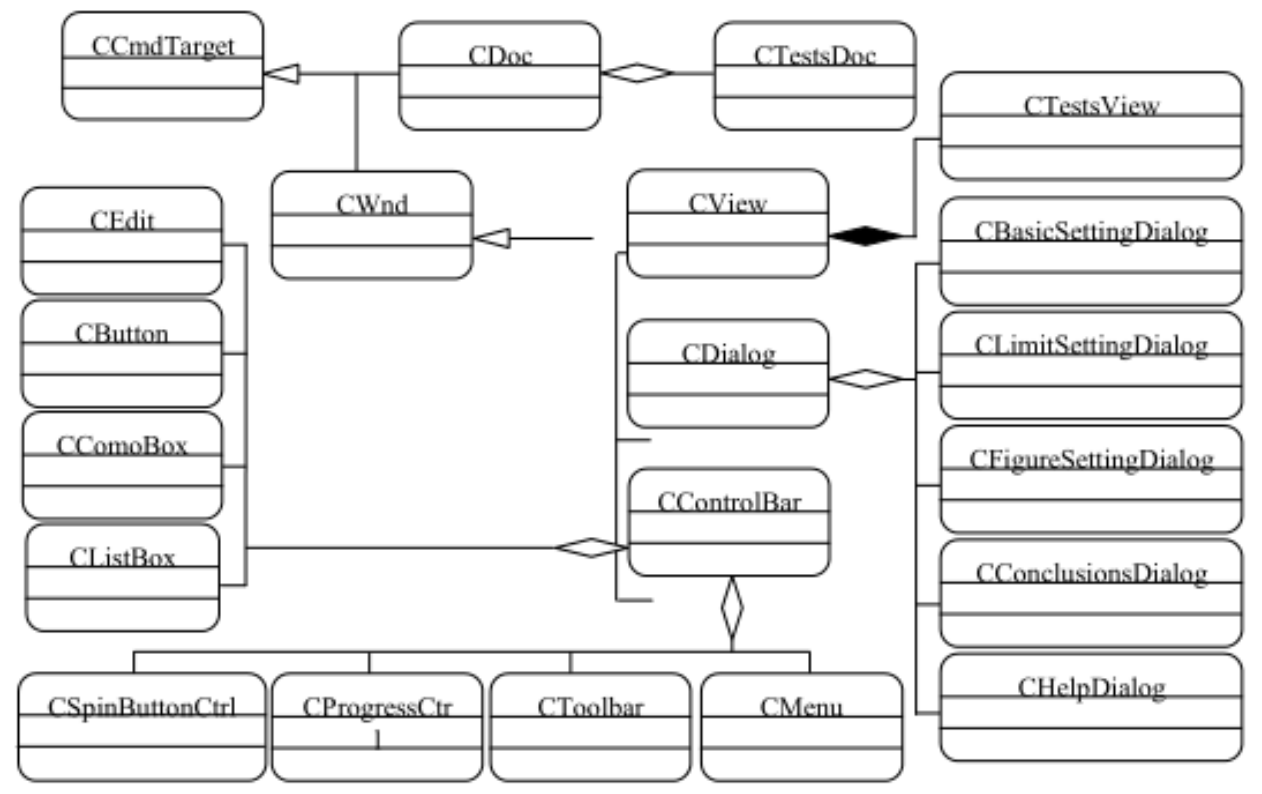

Figure 10 class analysis of object-oriented model 


\section{Example}

Research on integration technology of quality testing has been got some achievements including hardware and software, these are illustrated in figure 11. The research on integration technology of quality testing is focused on actual manufacturing factory, main aspects are: (a) factory incoming test, (b) process test include in-process test and on-line test, (c) finish test include dimension test and shape \& position test. Detail is shown in figure 11.

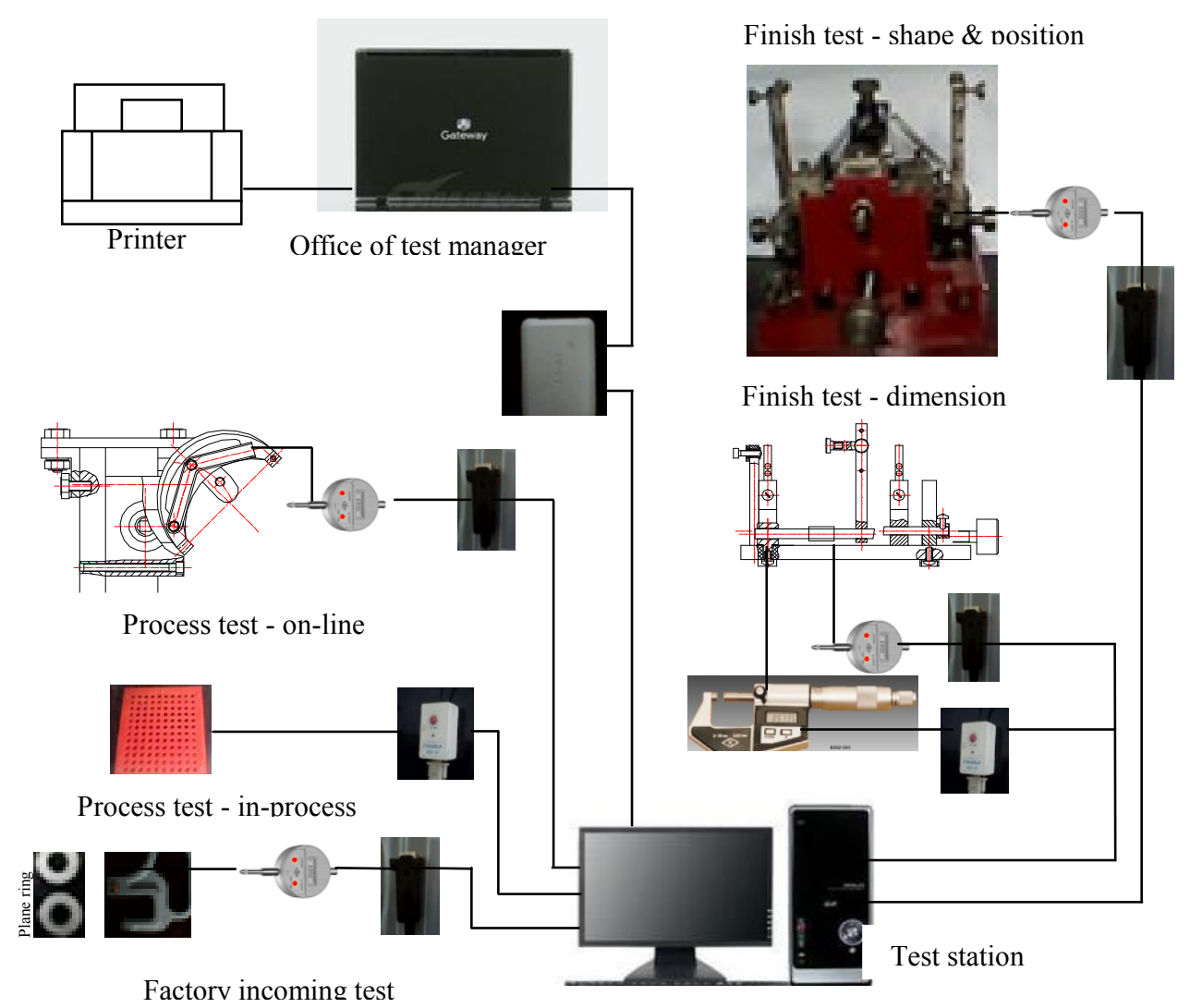

Figure 11 achievements illustration of integration technology of quality testing

\section{Conclusions}

In this research, we have investigated three aspects of integration technology of quality testing, including: (a) testing function integration, (b) equipment integration, and (c) computer integration. This paper is focused on integration of inspection equipment based on interface communication, that is the second aspect as above. RS232C, USB, and PCI are usual types for interface communication in quality testing, and an actual example is given with RS232C, in which, factory incoming test, process test, and finish test are addressed.

\section{References}

[1] T. Pfeifer, R. Freudenberg, L.Wiegers. Integrated Quality Control, Proceedings of the 4th Int. Symposium on Measurement Tech. And Intelligent Instruments, November 13-18, 1998, Hungary.

[2] Yang Kun, an application integration solution of B2B based on WIDL and SOAP, Xian Computer application, 2003,3. 
[3] Wang Hui, Design of wireless monitoring system based on Zigbee, Instrumentat and analysis monitor, 2012, 2.

[4] Wang Hui, Design of wireless transmiting system of airport data based on GPRS, Development and creativeness of mechanical and electric product, 2014, 3.

[5] Wang Huiying, Application of Image Recognition Technique to Quantity Examination for Machine Spare Parts, Xiandai machine, 2008,1:33-35.

[6] TanLi Mengqing, Development and simulated test of WEBSPC, IDDME \&ACCSPI2008, Beijing, Sept., 2008.

[7] A.Kusiak, T.Letsche and A.Zakarian. "Data modeling with IDEF1x, International Journal of Computer Integrated Manufacturing”, 1997, 10 (6): pp470-487. 